\title{
EL ECOTURISMO COMO MOTOR DE DESA- RROLLO EN ZONAS RURALES: UN ESTUDIO DE CASO EN REPÚBLICA DOMINICANA
}

Ecotourism as an engine of development in rural areas in developing countries. A case study in Dominican Republic

\section{Francisco Orgaz Agüera* Pablo Cañero Morales**}

Resumen: El turismo es uno de los principales sectores económicos, por lo que adquiere importancia en países en vías de desarrollo. De modo que conocer la percepción de la población local mejora el desarrollo del turismo en el lugar. El objetivo principal de esta investigación es conocer los beneficios económicos, socioculturales y medioambientales percibidos por el ecoturismo por parte de los residentes de comunidades rurales, en concreto, de aquellas localizadas en las proximidades al recurso natural y turístico Monumento Natural Saltos de la Damajagua (República Dominicana). Las principales hipótesis de esta investigación se basan en la idea de que la creación de empleo es uno de los cuatro principales beneficios mencionados por el turismo en espacios geográficos rurales; en que el turismo impulsa las actividades

Doctor en Turismo por la Universidad de Sevilla, España (2014). Es director ejecutivo de la Vicerrectoría de Investigación y Postgrado de la Universidad de UTESA, República Dominicana. Email: franciscoorgaz@docente.utesa.edu

** Tiene un máster en Dirección y Planificación del Turismo por la Universidad de Sevilla. Email: pablo_canero@hotmail.com 
culturales, revitalizando y conservando la herencia cultural de zonas rurales; $y$, en que el ecoturismo fomenta la conservación de los recursos naturales en áreas rurales. De todas ellas, en el Monumento Natural Saltos de la Damajagua se soporta la segunda, debido a que la primera no está dentro de los principales beneficios para los residentes, y la última, la percepción de la comunidad rural hacia la conservación del recurso natural es neutra. Aun así, los resultados obtenidos en esta investigación muestran que la población de estas zonas rurales tiene un cierto grado de acuerdo con todos los ítems planteados, sobre todo los relacionados con los beneficios económicos y socioculturales. Los resultados obtenidos en esta investigación pueden servir para que los gestores del Monumento Natural Saltos de la Damajagua conozcan cuáles son las valoraciones sobre el ecoturismo realizado en el área protegida a través del turismo comunitario. Sobre todo, se debe de mejorar las estrategias y políticas de conservación del área protegida, e invitar a los residentes a que formen parte de las mismas, con la finalidad de que estas conozcan que acciones se están desarrollando para mejorar la conservación del recurso.

Palabras clave: Ecoturismo, turismo comunitario, desarrollo local, comunidades rurales, República Dominicana.

Abstract: Tourism is a major economic sectors, with importance in developing countries. So, to know the perception of the local population enhances the development of tourism in the place. The main objective of this research is to understand the economic, cultural and environmental benefits from ecotourism perceived by residents of rural communities, in particular, those located in proximity to the natural and tourism resource of the Natural Monument Saltos Damajagua (Dominican Republic). The main hypothesis of this research has been based on that job creation is one of the four main benefits mentioned by tourism in rural geographic areas; that tourism promotes cultural activities, revitalizing and preserving the cultural heritage of rural areas; and in 
ecotourism promotes conservation of natural resources in rural areas. Of these, the Natural Monument Saltos Damajagua supports the second, because the first is not within the main benefits for residents, and the last, the perception of the rural community towards conservation of the natural resource is neutral. Even so, the results of this research show that the population of rural areas has a certain degree of agreement with all items raised, especially those related to the economic and cultural benefits. The results obtained in this research can serve to managers of Natural Monument Saltos Damajagua because this can know the valuations which are performed on ecotourism in the protected through community tourism area. Above all, it should improve the strategies and policies for the conservation of the protected area, and invite residents to be part of the same, in order to know that these actions are being developed to improve conservation.

Keywords: Ecotourism, Community based tourism, local development, rural communities, Dominican Republic.

\section{Introducción}

El turismo, sea cual sea su tipología, se configura como uno de los principales sectores económicos a nivel internacional (Ahmed y Krohn, 1992; Gursoy y Rutherford, 2004; Lasanta, 2010), si bien se han ido produciendo cambios significativos debido a que se ha pasado de un turismo de masas a un turismo alternativo que respeta el medio ambiente y donde el turista adquiere nuevas experiencias en contacto con la naturaleza, la cultura y la población local del destino turístico. En este sentido, en los últimos años han ido apareciendo nuevas formas de turismo más sostenibles, donde existen elementos relacionados con el descanso, el disfrute y la protección de la naturaleza o el conocimiento de la cultura, a través de acciones que buscan modelos sostenibles de desarrollo, debido sobre todo a 
que la sociedad está cada día más concienciada en temas relacionados con el impacto hacia el medio ambiente, tanto por motivos económicos como sociales, y cuya importancia, además, repercute en la calidad de vida de las comunidades locales, fomentando la reducción de la pobreza en las zonas rurales (WTO, 2002).

De acuerdo con López-Guzmán y Sánchez Cañizares (2009), la promoción del desarrollo turístico en zonas rurales de áreas en vías de desarrollo depende de diferentes factores de carácter social, político, económico y ambiental. En este sentido, el medio rural está experimentando transformaciones que rompen, por un lado, con la tradición productiva local y, por otro, con la estructura económica de las localidades, generando nuevas actividades económicas a través del turismo (Millán Vásquez de la Torre et al., 2011). De modo que las actividades turísticas realizadas en el medio rural deben ser respetuosas con el medio ambiente, natural y social, y estar basadas en los recursos naturales y culturales existentes en la zona geográfica (García, 2005).

El turismo adquiere una gran importancia para países en vías de desarrollo, debido que a través de este sector pueden mejorar el desarrollo socioeconómico de la población local (Gursoy y Rutherford, 2004; Dyer et al., 2007; Gu y Ryan, 2008). Y, sobre todo, en zonas rurales, donde el turismo se configura como una oportunidad para mejorar la calidad de vida de las comunidades locales, y por tal razón, en los últimos años ha aumentado las investigaciones sobre la importancia del turismo para el desarrollo de comunidades rurales (Lotero Contreras et al., 2008; LópezGuzmán y Sánchez Cañizares, 2009; Millán Vásquez de la Torre et al., 2011; Condesso, 2011; Gascón y Ojeda, 2014).

La teoría del intercambio social implica que el apoyo de la comunidad local al turismo depende de sus evaluaciones sobre los beneficios y costes generados por esta actividad (Andereck et al., 2005). Por consiguiente esta teoría proporciona una base conceptual para el examen de las interrelaciones entre las percepciones de los 
costes y beneficios, los impactos positivos y negativos y el apoyo al turismo (Ward y Berno, 2011). Por esta razón, últimamente son frecuentes las investigaciones que tienen por finalidad conocer la percepción que tiene la comunidad local hacia el turismo y los factores que influyen en dicha percepción. Según Pearce (1989) ha destacado la importancia que tiene la interacción entre turistas y residentes, reconociendo que el éxito y la sostenibilidad del turismo dependen del apoyo que recibe por parte de las comunidades locales, y por tanto, si no existe dicho apoyo el residente no se mostrará dispuesto a trabajar en este sector. Por esta razón es importante para los responsables políticos y empresarios conocer el apoyo de la comunidad local al turismo (Akis et al., 1996).

El objetivo principal de esta investigación es conocer los beneficios percibidos por el ecoturismo por parte de los residentes de comunidades rurales. El área de estudio se localiza en República Dominicana, en concreto en las comunidades de Imbert (Puerto Plata), donde se realiza una de las principales actividades complementarias al turismo de sol y playa: ecoturismo en el área natural protegida conocido como Monumento Natural Saltos de la Damajagua. Los beneficios analizados son de índole económica, sociocultural y medioambiental. Por otro lado, otros de los objetivos de este estudio es reforzar las investigaciones en el campo de la percepción de los residentes por el turismo en comunidades rurales. La elección de analizar solo los beneficios del turismo, y no los costes, viene dada por la importancia que estos tienen para mejorar la calidad de vida de los residentes (Pizam, 1978; Belisle y Hoy, 1980; Liu y Var, 1986; Gursoy y Rutherford, 2004; Dyer et al., 2007).

Para conseguir estos objetivos, este artículo se estructura tras esta introducción, en un segundo apartado donde se realiza una revisión de la literatura. En un tercer apartado se describe el área geográfica, para posteriormente, explicar la metodología de la investigación. 
En un quinto apartado se discuten los resultados del estudio, siguiéndole las conclusiones principales. Por último se muestran las referencias bibliográficas utilizadas por los autores.

\section{Revisión de la literatura}

A lo largo de los últimos años han aumentado las investigaciones por parte de la comunidad científica sobre los impactos del ecoturismo (Pickering y Hill, 2007; Deery et al., 2012; Ivanov y Webster, 2013; Kim et al., 2013), debido a que, actualmente, y según Hernández Martín (2004), uno de los principales temas de preocupación, entre los agentes sociales del turismo viene por establecer un marco conceptual y metodológico adecuado para analizar la actividad turística. Siguiendo a Andereck et al. (2005), en el análisis de la actitud del residente existen impactos positivos de al menos cuatro dimensiones: económica, social, cultural y medioambiental, y ambos pueden ser directos o indirectos, refiriéndose los primeros a los ingresos relacionados con el gasto realizado por los turistas y los segundos, a los gastos originados en la cadena de demanda de bienes y servicios.

En los últimos años, muchos han sido los investigadores y autores que han contribuido a la investigación del ecoturismo (Weaver y Lawton, 2007), desarrollándose análisis de la situación de esta tipología turística en diversas áreas geográficas. Hay que señalar que el concepto de ecoturismo ha sido definido por numerosos autores e investigadores, en ese orden de ideas Fennell (2001) ha identificado más de 85 definiciones de este concepto hasta el año 2001. Entre todas las definiciones de ecoturismo, una de las definiciones más completas, bajo nuestro punto de vista, es la de Jalani (2012) que considera que el ecoturismo hace referencia a aquel tipo de turismo que se desarrolla en áreas naturales y tiene como objetivo la conservación de las zonas naturales y el desarrollo de las comunidades locales. 
En cuanto a los componentes que conforman la actividad ecoturística, encontramos que el ecoturismo está formado por la demanda ecoturística que desarrollan actividades en contacto con la naturaleza; las actividades ecoturísticas como son las observaciones de aves o birdwatching, la observación de ballenas y tortugas marinas, recorridos por la selva, safaris fotográficos, buceo y snorkeling entre otras; los destinos ecoturísticos o aquellas áreas naturales protegidas que tienen gran belleza natural e interés ecológico; la infraestructura ecoturística que suele estar formada por las áreas recreativas, aulas de naturaleza, carril de ciclo-turismo, centro de visitantes, miradores, senderos y observatorios, entre otros; los guías de ecoturismo o personas encargadas de explicar los aspectos naturales y culturales del área natural a los ecoturistas; y los stakeholders del ecoturismo o todos aquellos actores que pueden afectar y ser afectados por las actividades del ecoturismo (Ingram y Durst, 1989; Fennel y Eagle, 1990; Williams, 1992; Puertas Cañaveral, 2007).

República Dominicana dispone de numerosas potencialidades ecoturísticas, pero existen diversas acciones que no se están desarrollando adecuadamente, entre las que destacan los inadecuados modelos de marketing de la actividad ecoturísticas, la promoción de las áreas protegidas, la creación de actividades ecoturísticas aprovechando el potencial de determinadas zonas del país o el establecimiento de una cuantía económica en la entrada de algunas áreas protegidas (Castellanos Verdugo y Orgaz Agüera, 2013), lo que podría generar recursos económicos que se reinvertirían en el mismo destino, tanto para mejorar la gestión del área natural como para generar nuevas oportunidades de negocio y empleo en la población local.

En este sentido, el turismo comunitario es una de las formas más adecuadas de planificar el ecoturismo en un destino, y en los últimos años, son muchos los países en vía de desarrollo los que están adoptando esta forma para explotar el turismo (Orgaz Agüera, 2013). Para Lopez-Gúzman y Sánchez Cañizares (2009), el turismo comunitario es una actividad que se fundamenta en la 
creación de productos turísticos bajo el principio básico de la necesaria participación de la comunidad local, y donde se pretende reducir el impacto negativo y reforzar los impactos positivos del turismo en la naturaleza (Casas Jurados et al., 2012). Por esta razón, Scheyvens (1999) sugiere que el término turismo comunitario se use únicamente para los casos donde los miembros de la población local tienen un alto control de las actividades y los beneficios. Para Ruiz et al. (2008), esta forma de turismo ha sido objeto de crítica por razones neocolonialistas, por su excesiva centralización en lo medioambiental, por su dependencia de las ONGs (Manyara et al., 2006), y por las deficiencias existentes desde la perspectiva del desarrollo de la comunidad local (Blackstock, 2005). Aunque, según Hiwaski (2006), este turismo tiene varios objetivos, destacando aquellos relacionados con la conservación de los recursos naturales, patrimoniales y culturales, el desarrollo socioeconómico de las comunidades locales y la calidad recibida por la demanda turística.

Siguiendo a López-Guzmán y Sánchez Cañizares (2009), el turismo comunitario se compone por tres bloques: el primero lo formarían los stakeholders encargados de la planificación turística (empresas, guías, gobierno local, oficinas de turismo, etc.); el segundo bloque lo conformarían las empresas de servicios directas (hoteles, restaurantes, etc.); y, por último, el tercer grupo estaría formado por otras empresas que apoyan el turismo en el destino (medios de transporte, tiendas de suvenir, turismo activo, etc.).

En definitiva, la puesta en marcha del ecoturismo a través del turismo comunitario fomenta el desarrollo socioeconómico de la población local (Manyara y Jones, 2007), y la conservación de la cultura y naturaleza local (López-Guzmán y Sánchez Cañizares, 2009) y funciona como una herramienta de lucha contra la pobreza (Casas Jurado et al., 2012). 


\section{Descripción del área geográfica}

Según Moreno Gil et al. (2002), el turismo en República Dominicana se caracteriza por un turista que visita el país para alojarse en hoteles-resort "todo incluido". Pero, siguiendo a García Sánchez y Alburquerque García (2003), el turismo de sol y playa debe adaptarse a las nuevas expectativas de los turistas y para ello se deben comercializar una serie de actividades complementarias a esa tipología de turismo, en el exterior de los hoteles, con la finalidad de mejorar los aspectos medioambientales, culturales y de hospitalidad de la comunidad local. Así, el turismo es la principal actividad económica de República Dominicana (Villarreal y Van Der Horst, 2008).

Para Agosín et al. (2009), República Dominicana tiene seis polos turísticos (figura n. ${ }^{\circ}$ ), que pasamos a desarrollar a continuación.

\section{Figura N. ${ }^{\circ} 1$}

Polos turísticos de República Dominicana

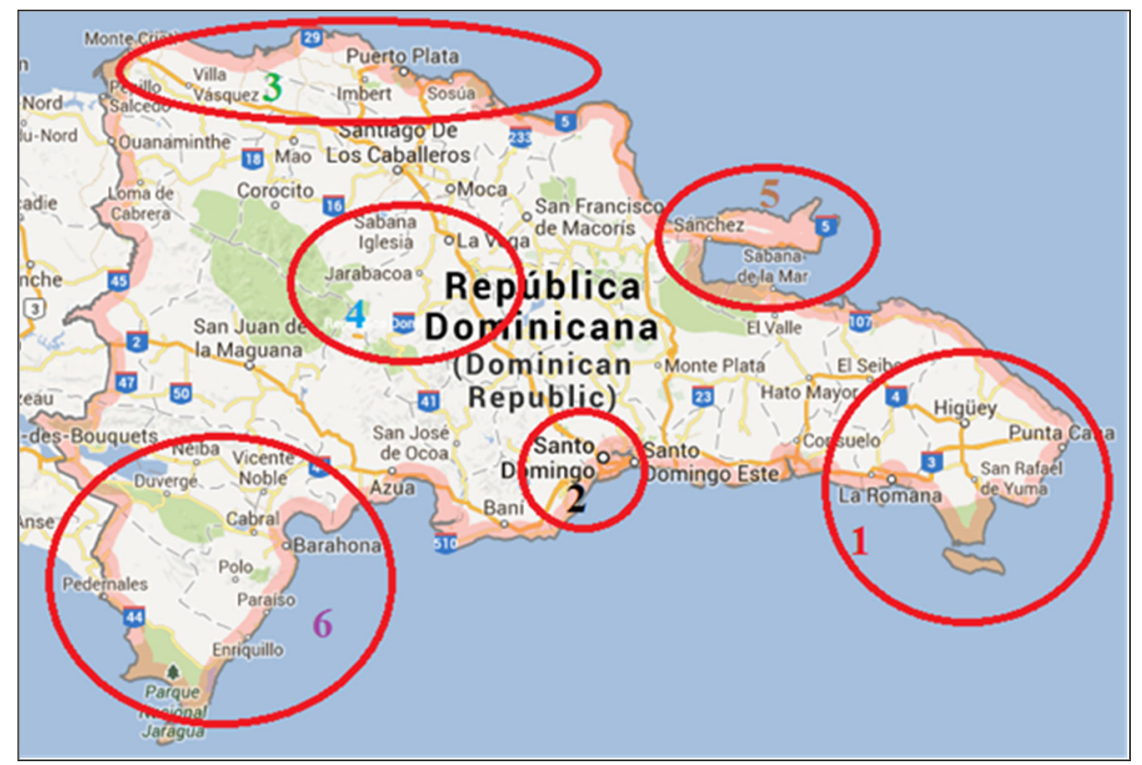

Fuente: Elaboración propia a partir de Google Maps. 
El Este de República Dominicana (número 1, figura n. ${ }^{\circ}$ 1) presenta el principal desarrollo turístico de sol y playa del país, con destinos consolidados a nivel mundial, como son Punta Cana, La Romana, Bayahibe y Bávaro. Además ahí se encuentra el área protegida más visitada del país, Parque Nacional del Este, beneficiándose de las excursiones que hacen los turistas de los hoteles-resort a la Isla Saona, comercializándose esta área desde finales del siglo pasado (Girault, 1998). La capital del país, Santo Domingo (número 2, figura $n .{ }^{\circ} 1$ ), se caracteriza por viajeros de negocios, lo que ha significado también un descenso del turismo de sol y playa en la zona de Boca Chica, principal playa de la capital (Agosín et al., 2009). Actualmente se configura como el segundo destino del país tras la zona este. Por otro lado, está la región norte y noroeste, donde destaca el desarrollo turístico de sol y playa y deportivo en Puerto Plata (número 3, figura n. ${ }^{\circ}$ ), en ocasiones beneficiando algunas otras provincia cercanas, menos desarrolladas turísticamente pero con gran potencial, como es el caso de Monte Cristi (Orgaz Agüera, 2014). Por su parte, en el centro del país están las ciudades de Constanza y Jarabacoa (número 4, figura n. ${ }^{\circ} 1$ ), donde se encuentran los principales destinos de montaña de República Dominicana (Agosín et al., 2009), destacando la zona más alta del país y de todo El Caribe: Pico Duarte. Otra zona turística se encuentra en la zona noreste, en concreto en la península de Samaná (número 5, figura n. ${ }^{\circ} 1$ ), donde existe un rápido crecimiento del turismo inmobiliario, además del propio turismo de sol y playa (Orgaz Agüera, 2014). También, y según Agosín et al. (2009), destaca el turismo de cruceros, consolidado desde la temporada 2005-2006. Por último, en la costa suroeste (número 6, figura n. ${ }^{\circ}$ 1) existe un enorme potencial ecoturístico, pero no está lo suficientemente desarrollado, si bien, empiezan a incorporarse pequeñas empresas locales que ofertan excursiones por las áreas protegidas (Agosín et al., 2009).

En lo relacionado con las áreas naturales, la Ley 202-04 de Áreas Protegidas del año 2004 clasifica en seis categorías de manejo las 
áreas protegidas de República Dominicana, formando parte todas ellas del Sistema Nacional de Áreas Protegidas (SINAP) del país (tabla n. ${ }^{\circ}$ ).

\section{Tabla N. ${ }^{\circ} 1$}

Categorías de manejo de área protegidas

\begin{tabular}{|l|l|}
\hline \multicolumn{1}{|c|}{ CATEGORÍA } & \multicolumn{1}{c|}{ SUBCATEGORÍA } \\
\hline I. Áreas de protección estrictas & $\begin{array}{l}\text { - Reserva científica } \\
\text { - Santuario de mamíferos marinos }\end{array}$ \\
\hline II. Parques nacionales & $\begin{array}{l}\text { - Parque nacional } \\
\text { - Parque nacional submarino }\end{array}$ \\
\hline III. Monumentos naturales & $\begin{array}{l}\text { - Monumento natural } \\
\text { - Monumento cultural }\end{array}$ \\
\hline $\begin{array}{l}\text { IV. Áreas de manejo de } \\
\text { hábitat/especies }\end{array}$ & - Refugio de vida silvestre \\
\hline $\begin{array}{l}\text { V. } \text { Reservas naturales } \\
\text { VI. Paisajes protegidos }\end{array}$ & $\begin{array}{l}\text { - Reservas forestales } \\
\text { - Reserva modelo }\end{array}$ \\
\hline & $\begin{array}{l}\text { - Vías panorivada } \\
\text { - Cómicas }\end{array}$ \\
\hline
\end{tabular}

Fuente: Elaboración propia.

Centrándonos en el recurso natural y turístico objeto de este estudio (Monumento Natural Saltos de la Damajagua), este forma parte de la categoría número tres de áreas protegidas del SINAP. Este recurso se aprovecha, principalmente, de las actividades complementarias de los turistas de sol y playa que visitan los hoteles-resort de la provincia de Puerto Plata. De modo que esta área protegida sigue en aumento en el número de visitas por año, lo cual ha generado más ingresos en las comunidades locales, consolidándose este recurso natural como un ejemplo de desarrollo sostenible para el país. 


\section{Metodología de la investigación}

El trabajo de campo de esta investigación ha sido dirigido a la población local que habita en las comunidades rurales de un recurso turístico natural de República Dominicana y que consideran el turismo como uno de los sectores que han favorecido el desarrollo socioeconómico de los residentes locales. La región concreta de este estudio ha sido las comunidades rurales de Imbert (Puerto Plata, al norte de República Dominicana), que se localiza en el tercer polo turístico del país. En concreto, las comunidades locales que aquí se localizan se benefician de la actividad turística del área protegida denominada Monumento Natural Saltos de la Damajagua.

El Monumento Natural Saltos de la Damajagua forma parte del Sistema Nacional de Áreas Protegidas de República Dominicana desde el 30 de junio de 2004. La extensión de esta área natural es de $6 \mathrm{~km}^{2}$ (600 hectáreas), albergando la cuenca del río que lleva su mismo nombre. Esta área protegida tiene una peculiaridad en cuanto a su manejo, debido a que es un recurso que se administra bajo la modalidad de co-manejo, entre diferentes organizaciones e instituciones: Ministerio de Medio Ambiente y Recursos Naturales; Asociación de Guías del Río Damajagua; Ministerio de Turismo; Gobernación provincial Puerto Plata; Ayuntamiento de Imbert; Asociación de Hoteles, condominios y establecimientos comerciales de Playa Dorada; la Administración del Monumento Natural; y los propietarios del terreno del Monumento Natural. Por tanto, el recurso se explota a través del turismo comunitario, debido a que son las comunidades locales quienes gestionan, planifican y obtienen los beneficios provenientes del área protegida. Los principales atractivos de esta zona natural son el río Damajagua, donde se realiza una caminata por el curso del río, sorteando las diferentes dificultades que ofrece el recorrido, en contacto con diferentes especies de flora y fauna, con equipos y guías. Además se ofertan varios servicios más, como restaurante buffet, bar, tiendas 
con productos artesanales, fotografías y vídeos y zapatos para la actividad. Este lugar está dotado con un Centro de Visitantes, baños, senderos y parking para los vehículos. Actualmente, el año 2013 cerró con más de 56.000 visitas, un 10\% más que el año anterior, consolidando el progreso constante de visitantes a esta área natural, que tiene como principal demanda un 30\% aproximadamente de excursionistas dominicanos y un $70 \%$ de turistas extranjeros (Heriberto Finke y Gómez Santana, 2014).

La metodología ha consistido en la técnica cuantitativa del cuestionario, utilizada para conocer las opiniones, percepciones y valoraciones sobre la sostenibilidad e impactos del turismo en las comunidades rurales. Esta técnica se ha realizado en las comunidades de Imbert, Puerto Plata, próximas al Monumento Natural Saltos de la Damajagua, y que se benefician del turismo de esta área protegida. El cuestionario fue presentado en español, tras la realización de un pre-test de 21 cuestionarios que ayudó a observar las posibles debilidades en las preguntas.

Con el fin de garantizar la validez del cuestionario, la estructura seguida se ha basado en estudios previos realizados en otros destinos por diferentes autores (Nicholas et al., 2009; Gursoy et al., 2009; Byrd et al., 2009; Gursoy et al., 2010; Lee, 2013). A partir de este conjunto inicial de ítems, se siguió un proceso de depuración de escalas en dos fases. Primero, dos investigadores especializados en turismo analizaron los ítems propuestos para cada constructo; y segundo, el cuestionario resultante fue revisado por un responsable del recurso turístico. De esta forma, se comprobó dos veces la validez. De modo que el cuestionario quedó formado por cinco partes diferenciadas:

1. Beneficios económicos del ecoturismo;

2. Beneficios sociales del ecoturismo;

3. Beneficios culturales del ecoturismo;

4. Beneficios medioambientales del ecoturismo; $y$, 
5. Perfil sociodemográficos de los encuestados. El número total de ítems es de 21.

En este sentido, las hipótesis de esta investigación son:

H1 La creación de empleo es uno de los cuatro principales beneficios mencionados por el turismo (Tyrrell y Spaulding).

H2 El turismo impulsa las actividades culturales, revitalizando y conservando la herencia cultural (Gilbert y Clark, 1997).

H3 El ecoturismo fomenta la conservación de los recursos naturales (Brandon, 1996).

El cuestionario se realizó entre los meses de marzo y abril de 2014 por parte de uno de los autores de esta investigación, que además tenía como misión explicar el propósito del estudio a los encuestados y responder las posibles preguntas del cuestionario que no comprendieran estos. En total, el número de cuestionarios válidos fue de 576 (tabla n. ${ }^{\circ}$ 2), obteniendo un error muestra del 4,03\% y un nivel de confianza del $95 \%$, teniendo en cuenta que el número de residentes es de 22.058 habitantes (Oficina Nacional de Estadística, 2010).

\section{Tabla N. ${ }^{\circ} 2$}

Ficha de la investigación

\begin{tabular}{ll}
\hline Población objetivo & 22.058 habitantes \\
\hline Ámbito geográfico & Provincia de Imbert (Puerto Plata, Rep. Dom.) \\
\hline Temporalidad & Marzo-Abril, 2014 \\
\hline Método de captación & Entrevista personal \\
\hline Procedimiento & Muestreo Aleatorio Simple \\
\hline Nivel de confianza & $95 \%$ \\
\hline Error muestral & $4,03 \%$ \\
\hline Cuestionarios válidos & 576 \\
\hline
\end{tabular}

Fuente: Elaboración propia. 
Las preguntas de las cuatro primeras secciones fueron respondidas a través de la Escala de Likert de cinco puntos. Las preguntas de la sección 5 eran cerradas con diferentes respuestas, y hacían referencia al perfil sociodemográfico del residente. El índice alfa de Cronbach es de 0,756. En este sentido, según Nunnally y Berstein (1994), se consideran aceptable una escala si su alfa de Cronbach está por encima de 0,7 .

Una vez cerrado el proceso de recepción de los cuestionarios se procedió a la informatización de los datos mediante la creación de una base de datos en el programa excel para su posterior utilización en los programas estadísticos IBM SPSS 22.

\section{Resultados de la investigación y discusión}

La tabla n. ${ }^{\circ} 3$ muestra las características sociodemográficas de los residentes. El 54,3\% de los encuestados han sido hombres, destacando un gran número de residentes en los grupos de edades de 35-44, 26-34 y de 18-25 años. El 41,3\% viven en un hogar con 3 miembros, y el $41,0 \%$ en una casa con 4 miembros. En cuanto a la actividad laboral, el 28,5\% trabajan por cuenta ajena, pero hay un grupo importante de personas desempleadas $(27,6)$.

Tabla N. ${ }^{\circ} 3$

Perfil sociodemográfico de los residentes

\begin{tabular}{lrcc}
\hline \multicolumn{2}{c}{ VARIABLE $(\mathbf{N}=\mathbf{5 7 6})$} & FRECUENCIA & PORCENTAJE (\%) \\
\hline Sexo & & & \\
& Hombre & 313 & 54,3 \\
& Mujer & 263 & 45,7 \\
\hline Edad & & & \\
& $18-25$ & 120 & 20,8 \\
& $26-34$ & 125 & 21,7 \\
& $35-44$ & 194 & 33,7 \\
& $45-54$ & 69 & 12,0 \\
& $55-64$ & 48 & 8,3 \\
& 65 o mas & 20 & 3,5 \\
\hline
\end{tabular}


VARIABLE $(\mathrm{N}=576)$

FRECUENCIA

PORCENTAJE (\%)

\section{Tamaño del hogar}

$\begin{array}{rcc}\text { Individual } & 2 & 0,3 \\ 2 \text { miembros } & 33 & 5,7 \\ 3 \text { miembros } & 238 & 41,3 \\ 4 \text { miembros } & 236 & 41,0 \\ 5 \text { o más miembros } & 67 & 11,6\end{array}$

\begin{tabular}{rcc}
\hline Actividad laboral & & \\
Estudiante & 105 & 18,2 \\
Trabajador cuenta propia & 70 & 12,2 \\
Trabajador cuenta ajena & 164 & 28,5 \\
Desempleado & 159 & 27,6 \\
Jubilado & 30 & 5,2 \\
Ama de casa & 48 & 8,3 \\
\hline
\end{tabular}

Fuente: Elaboración propia.

En la tabla n..$^{\circ} 4$ pueden observarse los beneficios económicos del turismo comunitario obtenidos por los residentes a través del ecoturismo. Según se muestra, la comunidad local apoyará el turismo según los beneficios que pueda percibir de la actividad (Nukoo y Ramkissoon, 2011). En este sentido, todos los ítems del cuestionario sobrepasan los cuatro puntos sobre cinco de la Escala de Likert, por lo que todos están de acuerdo en las afirmaciones de la tabla n. ${ }^{\circ} 4$. Por tanto, el ecoturismo mejora la situación económica de la comunidad rural, coincidiendo esto con otros estudios previos (Ahmed y Krohn, 1992; Gursoy y Rutherford, 2004). De acuerdo con Lindberg (1991) y Puerta Cañaveral (2007), el ecoturismo mejora la disposición de los gobiernos a participar en los proyectos turísticos y fomenta la autofinanciación de los recursos turísticos. Por su parte, el ecoturismo también proporciona nuevas oportunidades para invertir, sobre todo, a través de la creación de nuevos negocios locales (Davis et al., 1988; Kwan y McCartney, 2005), que a su vez, generan empleos (Dyer et al., 2007; Gu y Ryan, 2008). En este aspecto, según Tyrrell y Spaulding (1984), la creación de empleo es uno de los principales beneficios 
generados por el turismo para las comunidades rurales y en nuestra investigación ocupa el último lugar dentro de los beneficios económicos, por lo que la hipótesis H1 no es soportada en el Monumento Natural Saltos de la Damajagua.

\section{Tabla N. ${ }^{\circ} 4$}

\section{Beneficios económicos del ecoturismo percibidos por los residentes}

\begin{tabular}{|c|c|}
\hline ITEMS & MEDIA \\
\hline $\begin{array}{l}\text { El ecoturismo mejora la situación económica de la } \\
\text { comunidad local }\end{array}$ & 4,58 \\
\hline El ecoturismo genera ingresos al gobierno local & 4,44 \\
\hline El ecoturismo proporciona oportunidades de inversión & 4,39 \\
\hline El ecoturismo ayuda a la creación de negocios locales & 4,37 \\
\hline El ecoturismo fomenta el empleo en comunidades rurales & 4,28 \\
\hline Beneficios económicos totales & 4,41 \\
\hline
\end{tabular}

Fuente: Elaboración propia.

Por su parte, en la tabla n. 5 aparecen los beneficios sociales percibidos por la comunidad rural. Todos ellos sobrepasan la valoración media de cuatro puntos sobre cinco, por lo que todos están de acuerdo en la afirmación de cada ítem. Así, el ecoturismo fomenta la creación de nuevas empresas para las comunidades locales (Davis et al., 1988). Por su parte, genera actividades para que los residentes participen, fomentando así la cultura local (Puertas Cañaveral, 2007). De acuerdo con Ross y Wall (1999), el ecoturismo mejora los accesos hacia y entre las comunidades cercanas, y fomenta la construcción de nuevas infraestructuras y espacios de ocio, que buscan mejorar el turismo, pero también el bienestar de los residentes. Aunque, cabe destacar que según Tosun (2002), durante la temporada alta turística la población rural se sienten marginados de su comunidad. 


\section{Tabla N. ${ }^{\circ} 5$}

Beneficios sociales del ecoturismo percibidos por los residentes

\begin{tabular}{l|c}
\multicolumn{1}{c|}{ ITEMS } & MEDIA \\
\hline \begin{tabular}{l|l} 
El ecoturismo fomenta la creación de nuevas empresas \\
El ecoturismo genera actividades para que los residentes \\
participen
\end{tabular} & 4,41 \\
\hline $\begin{array}{l}\text { El ecoturismo mejora los accesos e infraestructura de la } \\
\text { comunidad }\end{array}$ & 4,40 \\
\hline $\begin{array}{l}\text { El ecoturismo genera más parques naturales de ocio } \\
\text { para el residente }\end{array}$ & 4,21 \\
\hline $\begin{array}{l}\text { El ecoturismo fomenta la preservación de la cultura local } \\
\text { Beneficios sociales totales }\end{array}$ & 4,15 \\
\hline \hline
\end{tabular}

Fuente: Elaboración propia.

En otro sentido, la tabla n. ${ }^{\circ} 6$ muestra los beneficios culturales obtenidos por la población residente. Así, se puede observar que el ecoturismo fomenta la conservación de la herencia cultural de las comunidades locales (Gilbert y Clark, 1997), por lo que la hipótesis H2 de esta investigación es soportada en el Monumento Natural Saltos de la Damajagua. Además, el ecoturismo facilita el acceso a la comunidad rural a las actividades culturales (Dyer et al., 2007; Stronza y Gordillo, 2008), generando también un impacto positivo en la identidad local y facilitando el intercambio cultural entre el residente y el turista (Besculides et al., 2002). 


\section{Tabla N. ${ }^{\circ} 6$}

\section{Beneficios culturales del ecoturismo percibidos por los residentes}

\begin{tabular}{|c|c|}
\hline ITEMS & MEDIA \\
\hline $\begin{array}{l}\text { El ecoturismo fomenta la conservación de la herencia } \\
\text { cultural }\end{array}$ & 4,36 \\
\hline $\begin{array}{l}\text { El ecoturismo facilita el acceso del residente a actividades } \\
\text { culturales }\end{array}$ & 4,32 \\
\hline $\begin{array}{l}\text { El ecoturismo tiene un impacto positivo en la identidad } \\
\text { local }\end{array}$ & 4,31 \\
\hline $\begin{array}{l}\text { El ecoturismo facilita el intercambio cultural entre locales } \\
\text { y turistas }\end{array}$ & 4,26 \\
\hline Beneficios culturales totales & 4,31 \\
\hline
\end{tabular}

Fuente: Elaboración propia.

Por su parte, en la tabla n. ${ }^{\circ} 7$ se muestran los beneficios medioambientales del ecoturismo obtenidos por la población rural. Así, los ítems analizados tienen una valoración neutra, oscilando entre "no estoy de acuerdo" y "estoy de acuerdo". Por tanto, la hipótesis $\mathrm{H} 3$, que afirmaba que el ecoturismo fomenta la conservación de los recursos naturales (Brandon, 1996) no es soportada por el Monumento Natural Saltos de la Damajagua. Así, tampoco se considera que propicie la creación de nuevas áreas protegidas y la mejora de la infraestructura local. En este sentido, cabe destacar que el impacto ambiental del turismo se suele relacionar con las repercusiones de las actividades turísticas en el medio natural y/o rural, con especial referencia a la capacidad de carga física de un recurso, concepto que hace referencia al máximo número de personas que pueden utilizar un emplazamiento sin provocar una alteración irreversible en el entorno natural/rural y sin un declive inaceptable en la calidad de la experiencia de los visitantes (Mathieson y Wall, 1982). 
Tabla N. ${ }^{\circ} 7$

Beneficios medioambientales del ecoturismo percibidos por los residentes

\begin{tabular}{|c|c|}
\hline ITEMS & MEDIA \\
\hline $\begin{array}{l}\text { El ecoturismo genera recursos para conservar el área } \\
\text { protegida }\end{array}$ & 3,15 \\
\hline $\begin{array}{l}\text { El ecoturismo propicia la creación de nuevas áreas } \\
\text { protegidas }\end{array}$ & 3,12 \\
\hline $\begin{array}{l}\text { El ecoturismo genera una mejora de la infraestructura } \\
\text { local }\end{array}$ & 3,04 \\
\hline Beneficios medioambientales totales & 3,10 \\
\hline
\end{tabular}

Fuente: Elaboración propia.

En definitiva, los resultados obtenidos en las tablas anteriores muestran que los valores medios de los ítems están comprendidos entre 3 y 5, lo que nos indica que, en general, los participantes de esta investigación muestran un cierto grado de acuerdo con todos los ítems planteados. Cabe destacar que los beneficios económicos $(4,41)$ son los que más perciben las comunidades rurales, seguidos de los beneficios socioculturales $(4,31)$. Por su parte, los beneficios medioambientales $(3,10)$ son los que tienen la percepción más baja para los residentes. Por tanto, existe una implicación de la población rural con el recurso natural y turístico Monumento Natural Saltos de la Damajagua.

\section{Conclusiones}

El objetivo principal de esta investigación es conocer los beneficios económicos, socioculturales y medioambientales percibidos por el ecoturismo por parte de los residentes de comunidades rurales, en concreto, de aquellas localizadas en las proximidades al recurso natural y turístico Monumento Natural Saltos de la Damajagua. 
Las principales hipótesis de esta investigación se han basado en que la creación de empleo es uno de los cuatro principales beneficios mencionados por el turismo en espacios geográficos rurales; en que el turismo impulsa las actividades culturales, revitalizando y conservando la herencia cultural de zonas rurales; y, en que el ecoturismo fomenta la conservación de los recursos naturales en áreas rurales. De todas ellas, en el Monumento Natural Saltos de la Damajagua se soporta la segunda, debido a que la primera no está dentro de los principales beneficios para los residentes, y la última, la percepción de la comunidad rural hacia la conservación del recurso natural es neutra. Aun así, los resultados obtenidos en esta investigación muestran que la población de estas zonas rurales tiene un cierto grado de acuerdo con todos los ítems planteados, sobre todo los relacionados con los beneficios económicos y socioculturales.

Los resultados obtenidos en esta investigación pueden servir para que los gestores del Monumento Natural Saltos de la Damajagua conozcan cuáles son las valoraciones sobre el ecoturismo realizado en el área protegida a través del turismo comunitario, por lo que debe mejorar las estrategias y políticas de conservación del área protegida e invitar a los residentes a que formen parte de las mismas, con la finalidad de que estas conozcan qué acciones se están desarrollando para mejorar la conservación del recurso.

La principal limitación de este estudio viene dada porque la investigación está planteada desde el punto de vista de la población rural, dificultando la difusión de los descubrimientos a otros agentes locales relevantes para el turismo, ya sea de forma directa $\mathrm{o}$ indirecta.

Entre las futuras líneas de investigación está desarrollar este estudio en otros destinos ecoturísticos del país (Parque Nacional Los Haitises y Parque Nacional del Este), con la finalidad de comparar los resultados obtenidos en ambos recursos, y poder analizar de manera global la percepción de los residentes dominicanos en 
torno a ecoturismo y sus beneficios para las comunidades rurales próximas. Hay que decir que otra línea de investigación sería adaptar este estudio a otros sectores, como la agricultura, ya que se configura como unos de los sectores más importantes en el país junto al turismo.

\section{Bibliografía}

Agosín, M.; Manzano, O.; Rodríguez-Balza, R. \& Stein, E. (2009). La ruta bacia el crecimiento sostenible en la República Dominicana: Fiscalidad, competitividad, institucionalidad y electricidad. Santo Domingo: Banco Interamericano de Desarrollo.

Ahmed, Z. U. \& Krohn, F. B. (1992). Marketing India as a tourist destination in North America-challenges and opportunities. International Journal of Hospitality Management, 11(2), 89-98.

Akis, S.; Persistianis, N. \& Warner, J. (1996). Resident's attitudes to tourism development: the case of Cyprus. Tourism Management, 17(7), 481-494.

Andereck, K. L.; Valentine, K. M.; Knopf, R. C. \& Vogt, C. A. (2005). Resident's perceptions of community tourism impacts. Annals of Tourism Research, 32(4), 1056-1076.

Belisle, F. J. \& Hoy, D. R. (1980). The perceived impact of tourism by residents: A case study in Santa Maria, Columbia. Annals of Tourism Research, 7(1), 83-101.

Besculides, C. A.; Lee, M. E. Mc. \& Cormick, P. J. (2002). Resident's perceptions of the cultural benefits of tourism. Annals of Tourism Research, 29(2), 303-319.

Blackstock, K. (2005). A critical look at community based tourism. Community Development Journal, 40(1), 39-49. 
Brandon, K. (1996). Ecotourism and conservation: A review of key issues. Washington DC: World Bank.

Byrd, E. T.; Bosley, H. E. \& Dronberger, M. G. (2009). Comparisons of stakeholder percpetions of tourism impacts in rural eastern North Carolina. Tourism Management, 30(5), 693-703.

Casas Jurado, A. C.; Soler Domingo, A. \& Jaime Pastor, V. (2012). El turismo comunitario como instrumento de erradicación de la pobreza: Potencialidades para su desarrollo en Cuzco (Perú). Cuadernos de Turismo, 30, 91-108.

Castellanos Verdugo, M. \& Orgaz Agüera, F. (2013). Potencialidades ecoturísticas de la República Dominicana. TURyDES: Revista de Investigación en Turismo y Desarrollo Local, 6(14), 1-9.

Condesso, F. (2011). Desenvolvimento rural, patrimonio e turismo. Cuadernos de Desarrollo Rural, 8(66), 197-222.

Davis, D.; Allen, J. \& Cosenza, R. M. (1988). Segmenting local residents by their attitudes interests and opinions toward tourism. Journal of Travel Research, 27(2), 2-8.

Deery, M.; Jago, L. \& Fredline, L. (2012). Rethinking social impacts of tourism research: A new research agenda. Tourism Management, 33(1), 64-73.

Dyer, P.; Gursoy, D.; Sharma, B. \& Carter, J. (2007). Structural modeling of resident perceptions of tourism and associated development on the Sunshine Coast, Australia. Tourism Management, 28, 409-422.

Fennell, D. A. (2001). A content analysis of ecotourism definitions. Current Issues in Tourism, 4, 403-421.

Fennell, D. A. \& Eagles, P. (1990). Ecotourism in Costa Rica: A conceptual framework. Journal Park and Recreation Administration, 1(1), 23-34. 
García, B. (2005). Características diferenciales del producto turismo rural. Cuadernos de Turismo, 15, 113-133.

García Sánchez, A. \& Alburquerque García, F. J. (2003). El turismo cultural y el de sol y playa: ¿Sustitutivos o complementarios? Cuadernos de Turismo, 11, 97-105.

Gascón, J. \& Ojeda, D. (2014). Turistas y campesinado. El turismo como vector de cambio de las economías campesinas en la era de la globalización. Madrid/El Sausal (Tenerife): PASOS Edita.

Gilbert, D. \& Clark, M. (1997). An exploratory examination of urban tourism impact, with reference to resident's attitudes, in the cities of Canterbury and Guildford. Cities, 14(6), 343-352.

Girault, C. (1998). El auge del turismo en la República Dominicana: Implicación social y política. Ciencia y Sociedad, 23(3), 417-426.

Gu, H. \& Ryan, C. (2008). Place attachment, identity and community impacts of tourism - The case of Beijing Hutong. Tourism Management, 29, 637-647.

Gursoy, D. \& Rutherford, D. G. (2004). Host attitudes toward tourism: An improved structural model. Annals of Tourism Research, 31, 495-516.

Gursoy, D.; Chi, C. G. \& Dyer, P. (2009). An examination of local's attitudes. Annals of Tourism Research, 36, 723-726.

Gursoy, D.; Chi, C. G. \& Dyer, P. (2010). Local's attitudes toward mass and alternative tourism: The case of Sunshine Coast, Australia. Journal of Travel Research, 49, 381-394.

Heriberto Finke, C. \& Gómez Santana, R. (2014). Espacios naturales protegidos de la provincia de Puerto Plata. Puerto Plata: Ministerio de Medio Ambiente y Recursos Naturales. 
Hiwasaki, L. (2006). Community-based tourism: A pathway to sustainability for Japan's protected areas. Society and Natural Resources, 19(8), 675-692.

Ingram, C. D. \& Durst, P. B. (1989). Nature oriented tour operators: Travel to developing countries. Journal Travel Research, 2(28), 11-15.

Ivanov, S. \& Webster, C. (2013). Tourism's impact on growth: The Role of Globalisation. Annals of Tourism Research, 41, 231-236.

Jalani, J. O. (2012). Local People's Perception on the Impacts and Importance of Ecotourism in Sabang, Palawan, Philippines. Procedia: Social and Behavioral Sciences, 57, 247-254.

Kim, K.; Uysal, M. \& Sirgy, M. J. (2013). How does tourism in a community impact the quality of life of community residents? Tourism Management, 36, 527-540.

Kwan, F. V. C. \& McCartney, G. (2005). Mapping resident's perceptions of gaming impacts. Journal of Travel Research, 44, 177-187.

Lee, T. H. (2013). Influence analysis of community resident support for sustainable tourism development. Tourism Management, 34, 37-46.

Lasanta, T. (2010). El turismo de nieve como estrategia de desarrollo en el pirineo aragonés. Cuadernos de Investigación Geográfica, 36(2), 145-163.

Lindberg, K. (1991). Policies for maximizing nature tourism's ecological and economics benefits. Washington, DC: World Resources Institute.

Liu, J. C. \& Var, T. (1986). Residential attitudes toward tourism impact in Hawai. Annals of Tourism Research, 13(2), 193-214. 
López-Guzmán, T. \& Sánchez Cañizares, S. M. (2009). Desarrollo socioeconómico de las zonas rurales con base en el turismo comunitario. Un estudio de caso. Cuadernos de Desarrollo Rural, 6(62), 81-97.

Lotero Contreras, J.; Arcila, M. \& Gómez Vargas, A. B. (2008). Políticas públicas y turismo en las regiones rurales de Antioquia, Colombia: aproximaciones desde el enfoque de sistemas productivos territoriales y redes institucionales. Cuadernos de Desarrollo Rural, 5(61), 169-193.

Manyara, G. \& Jones, E. (2007). Community-based tourism enterprises development in Kenya: An exploration of their potential as avenues of poverty reduction. Journal of Sustainable Tourism, 15(6), 628-644.

Manyara, G.; Jones, E. \& Botterill, D. (2006). Tourism and poverty alleviation: The case for indigenous enterprise development in Kenya. Tourism, Culture and Communication, 7(1), 19-37.

Mathieson, A. \& Wall G. (1982). Tourism: Economic, Physical, and Social Impacts. New York: Longman House.

Millán Vázquez de la Torre, M. G.; Agudo Gutiérrez, E. M. \& Morales Fernández, E. (2009): Análisis de la oferta y la demanda de oleoturismo en el sur de España: un estudio de caso. Cuadernos de Desarrollo Rural, 8(67), 181-202.

Moreno Gil, S.; Celis Sosa D. F. \& Aguilar Quintana, T. (2002). Análisis de la satisfacción del turista de paquetes turísticos respecto a las actividades de ocio en el destino: El caso de República Dominicana. Cuadernos de Turismo, 9, 67-84.

Nicholas, L.; Thapa, B. \& Ko, Y. (2009). Resident's perspectives of a World Heritage site - The Pitons Management Area, St. Lucia. Annals of Tourism Research, 36(3), 390-412. 
Nunnally, J. \& Bernstein, I. (1994). Psychometric Theory. New York: McGraw-Hill.

Orgaz Agüera, F. (2013). El turismo comunitario como herramienta para el desarrollo sostenible de destinos subdesarrollados. Nómadas, Revista Crítica de Ciencias Sociales y Jurídicas, 38, 1-13.

Orgaz Agüera, F. (2014). El ecoturismo en los humedales: Análisis de las potencialidades de República Dominicana. Rosa dos Ventos, 6(1), 4-18.

Pearce, P. L. (1994). Tourist-resident impact: Examples and emerging solutions. En W. F. Theobald (Ed.). Global tourism: the next decade (pp. 103-123). Oxford: ButterworthHeinemann.

Pickerin, C. M. \& Hill, W. (2007). Impacts of recreation and tourism on plant biodiversity and vegetation in protected areas in Australia. Journal of Environmental Management, 85(4), 791-800.

Pizam, A. (1978). Tourism's Impacts: The Social Costs to the Destination Community as Perceived by its Residents. Journal of Travel Research, 16(4), 812.

Puertas Cañaveral, I. (2007). Ecoturismo en las Reservas de la Biosfera. Granada: Universidad de Granada.

Ross, S. \& Wall, G. (1999). Ecotourism: towards congruence between theory and practice. Tourism Management, 20(1), 123-132.

Ruiz, E.; Hernández, M.; Coca, A.; Cantero, P. \& Del Campo, A. (2008). Turismo comunitario en Ecuador. Comprendiendo el community-based tourism desde la comunidad. PASOS: Revista de Turismo y Patrimonio Cultural, 6(3), 399-418.

Scheyvens, R. (1999). Ecotourism and the Empowerment of Local Communities. Tourism Management, 20, 245-49. 
Stronza, A. \& Gordillo, J. (2008). Community views of ecotourism. Annals of Tourism Research, 35(2), 448-468.

Tosun, C. (1998). Roots of unsustainable tourism development at the local level: the case of Ürgüp in Turkey. Tourism Management, 19, 595-610.

Tyrrell, T. \& Spaulding P. (1984). A survey of attitudes toward tourism growth in Rhode Island. Hospitality Education and Research Journal, 8, 22-23.

Villareal, R. \& Van Der Horst, A. (2008). Estrategia de competitividad turística de la República Dominicana. ARA, Journal of Tourism Research, 1(1), 15-28.

Ward, C. \& Berno, T. (2011). Beyond social exchange theory. Annals of Tourism Research, 38(4), 1556-1569.

Weaver, D. B. \& Lawton, L. J. (2007). Twenty years on: The state of contemporary ecotourism research. Tourism Management, 28(5), 1168-1179.

Williams, P. (1992). A Local Framework for Ecotourism Development. Western Wildlands, 18(3), 14-19.

World Tourism Organization (2002). Tourism and Proverty Alleviation. Madrid: World Tourism Organization. 


\section{Francisco Orgaz Agüera}

Es doctor en Turismo por la Universidad de Sevilla, España (2014), espacio en el que tuvo la oportunidad de analizar la actividad ecoturística de República Dominicana; diplomado en Turismo por la Universidad de Córdoba, España (20072010); máster en Dirección y Planificación del Turismo por la Universidad de Sevilla, España (2010-2011). Actualmente imparte docencia en la Maestría en Administración de Empresas y en la Licenciatura en Administración de Empresas Turísticas de la Universidad Tecnológica de Santiago (UTESA), República Dominicana. Es director Ejecutivo de la Vicerrectoría de Investigación y Postgrado de la Universidad de UTESA.

Autor de más de 50 artículos en revistas de investigación y congresos y autor de dos libros. Sus principales líneas de investigación son el turismo y el desarrollo sostenible. Es co-director de la revista cientificas International of Journal of World of Tourism, dirigida por la Asociación MUTUS WORLD de la Facultad de Turismo y Finanzas de la Universidad de Sevilla. Email: franciscoorgaz@docente.utesa.edu

\section{Pablo M. Cañero Morales}

$\mathrm{Ph}(\mathrm{c}) \mathrm{H}$ por la Universidad de Córdoba, España; máster en Dirección y Planificación del Turismo por la Universidad de Sevilla, en la especialidad de dirección de empresas; diplomado en Turismo por la Universidad de Córdoba. 
Actualmente cursa el Máster Interuniversitario en Sostenibilidad y Responsabilidad Social Corporativa de la Universidad Nacional de Educación a Distancia (UNED) y la Universidad Jaume I de Castellón.

Es investigador en Turismo y asesor en la Asociación Internacional para el Desarrollo de Iniciativas Sostenibles. Ha publicado diversos artículos en revistas científicas y congresos. También es editor de la revista científica International Journal of World of Tourism de la Asociación Mutus World.

Email: pablo_canero@hotmail.com

Recibido: 09/09/2014 Aprobado: 24/02/2015

76 Ciencia y Sociedad 2015; 40(1): 47-76 